\title{
Efecto de las barreras de acceso sobre la asistencia a citas de programa de control prenatal y desenlaces perinatales ${ }^{*}$
}

\section{Effect of the Access Barriers on Attending to Pregnant Control Appointments and its Perinatal Outcomes}

\section{Efeito das barreiras de acesso a comparecimento a consultas médicas programadas de controle pré-natal e desenlaces perinatais}

Fecha de recepción: 26-02-14 Fecha de aceptación: 02-04-14 Disponible en línea: 01-07-14 doi: 10.11144/Javeriana.rgyps13-27.ebas

Cómo citar este artículo:

Rodríguez-Páez FG, Jiménez-Barbosa WG, Jiménez-González CA, Coral-Córdoba AE, Ramírez-Solano PC, Ramos-Navas NR. Efecto de las barreras de acceso sobre la asistencia a citas de programa de control prenatal y desenlaces perinatales. Rev. Gerenc. Polít. Salud. 2014; 13(27): 212-227. http://dx.doi. org/10.11144/Javeriana.rgyps13-27.ebas

\author{
Fredy Guillermo Rodríguez-Páez** \\ Wilson Giovanni Jiménez-Barbosa*** \\ Cindy Alejandra Jiménez-González**** \\ Ángela Edith Coral-Córdoba***** \\ Paola Carolina Ramírez-Solano****** \\ Néstor Raúl Ramos-Navas*******
}

\footnotetext{
Artículo de investigación realizado por estudiantes y docentes de la Universidad Jorge Tadeo Lozano, sede Santa Marta, resultado del trabajo de grado titulado Efecto de la identificación e intervención de barreras de acceso en la asistencia a citas de programa de control prenataly desenlaces perinatales. Entidad financiadora: sin financiamiento directo; apoyo de contrapartida en especie de la Universidad Jorge Tadeo Lozano; número de contrato: sin contrato. Fecha de iniciación: 21 de febrero de 2012; finalización: 29 de noviembre 29 de 2013.

** Magíster en Salud Pública de la Universidad de Antioquia. Coordinador académico del Área de Postgrados en Gestión de Servicios de Salud y Seguridad Social, Universidad de Bogotá Jorge Tadeo Lozano, Calle 23 No. 3-19, módulo 16 (posgrados) oficina 301, código postal 110010, Bogotá, D.C. Colombia. Correo electrónico: fredyg.rodriguezp@utadeo.edu.co

*** Doctor en Ciencias Sociales, Niñez y Juventud de la Universidad de Manizales-Cinde. Docente asociado II del Área de Gestión de Servicios de Salud y Seguridad Social, Universidad de Bogotá Jorge Tadeo Lozano. Correo electrónico: wilsong. jimenezb@utadeo.edu.co

***** Especialista en Gerencia y Auditoría de la calidad en salud de la Universidad de Bogotá Jorge Tadeo Lozano. Gerente de la IPS ADM, sede Santa Marta. Correo electrónico: cindya.jimenezg@utadeo.edu.co

**s*** Especialista en Gerencia y Auditoría de la calidad en salud de la Universidad de Bogotá Jorge Tadeo Lozano. Clínica Dentalium, sede Santa Marta. Correo electrónico: angelacoral84@yahoo.es

******* Especialista en Gerencia y Auditoría de la calidad en Salud Universidad de Bogotá Jorge Tadeo Lozano. Bacterióloga, Clínica

212 de la Mujer, sede Santa Marta. Correo electrónico: pcrs311@ @otmail.com Tadeo Lozano, sede Santa Marta. Correo electrónico: nestorr.ramosn@utadeo.edu.co
} 


\section{Resumen}

Objetivo: determinar qué condiciones pueden explicar la inasistencia a citas programadas de control prenatal (CPN) y evaluar si la eliminación de las barreras de acceso mejora las condiciones de salud de las gestantes. Metodología: se realizó un estudio de diseño mixto, multietápico, de cohorte prospectivo. Resultados: la dificultad para el pago de transporte fue la principal barrera de acceso causante de inasistencia. Mediante la entrega de subsidios de transporte, las inasistencias disminuyeron $87 \%$. Los determinantes de la salud asociados con mayor probabilidad de presentar complicaciones perinatales fueron: estrato socioeconómico bajo, distancia de la vivienda respecto al sitio de atención y alta puntuación en escala de riesgo biopsicosocial. Adicionalmente, factores como educación superior y sustento económico a cargo del hombre cabeza de familia se asociaron a reducción del riesgo de complicaciones materno-fetales. Conclusiones: las barreras económicas, socioculturales y geográficas, obviadas por el sistema de salud colombiano, afectan la accesibilidad al CPN.

Palabras clave: mujeres embarazadas; atención prenatal; prestación de atención de salud; determinantes sociales de la salud; accesibilidad a los servicios de salud; apoyo a la planificación en salud

\section{Abstract}

Objective: To determine conditions that may explain the failure to attend the programmed prenatal control appointments (CPN), and assessing whether eliminating the access barriers improves the health conditions of pregnant women. Methodology: We performed a mixed design prospective cohort study in several stages. Results: The main access barrier that caused failures to attend was difficulties to pay for transportation. By giving transportation subsidies, the failures to attend to the appointments were reduced by $87 \%$. The health determinants associated with a higher probability of having perinatal complications were: low socio-economic status, distance from their residence to the attention site, and a high score in the bio-psychosocial risk scale. Additionally, factors like tertiary education and economic support in charge of the man head of the household were associated with a decrease in the risk of maternal-fetal complications. Conclusions: the economic, socio-cultural and geographical barriers ignored by the Colombian health system affect access to the CPN.

Keywords: pregnant women; prenatal attention; health care provision; social determinants of health; accessibility to health services; support to health planning

\section{Resumo}

Objetivo: determinar quais condições podem explicar o não comparecimento às consultas médicas programadas de controle pré-natal (CPN) e avaliar se a remoção das barreiras de acesso melhora as condições de saúde das gestantes. Metodologia: realizou-se estudo de desenho misto, multiestágio, de coorte prospectivo. Resultados: a dificuldade para o pagamento de transporte foi a principal barreira de acesso causante do não comparecimento. Ao fornecer subsídios de transporte, os não comparecimentos diminuíram $87 \%$. Os determinantes da saúde associados com maior probabilidade de apresentar complicações perinatais foram: baixo estrato socioeconómico, distância da moradia respeito do local de atendimento e alta pontuação em escala de risco biopsicosocial. Aliás, fatores como ensino superior e sustento económico a cargo do homem cabeça de família foram associados a redução de risco de complicações materno-fetais. Conclusões: as barreiras económicas, socioculturais e geográficas, obviadas pelo sistema de saúde colombiano, afetam a acessibilidade ao CPN

Palavras-chave: mulheres grávidas, atendimento pré-natal; prestação de atendimento de saúde; determinantes sociais da saúde; acessibilidade aos serviços de saúde; apoio e planejamento em saúde 


\section{Introducción}

En Colombia, la Constitución de 1991 no reconoció la salud como un derecho fundamental, sino como un servicio público cuya garantía es responsabilidad del Estado, teniendo como principios la universalidad, la eficiencia y la solidaridad (1). La comprensión como derecho fundamental advino cuando la Corte Constitucional, mediante análisis de diversos amparos de tutela, hizo conexa la salud con el derecho fundamental a la vida (2).

El contrato social definido por la Constitución de 1991, en lo referente a salud fue reglamentado en 1993, mediante la promulgación de la Ley 100, la que en su libro segundo creó el Sistema General de Seguridad Social en Salud (sGsss). Dicho sistema establece las pautas generales de financiación, organización y prestación de servicios de salud (3).

Dentro de los principios establecidos para el SGSss se incorpora el de equidad, la que es entendida "como la igualdad de oportunidades que deben tener todas las personas para alcanzar su pleno potencial de salud sin desventaja, si ello puede evitarse" (4). La equidad es materializada mediante el financiamiento público del sistema, a través de recursos parafiscales y de tributación general, y por medio del acceso universal a un plan unificado de beneficios denominado Plan Obligatorio de Salud (POS). De esta manera, se busca la eliminación de las desigualdades en la prestación de servicios de salud, promoviendo condiciones de acceso para toda la población del territorio nacional en todos los niveles de atención.

Para la puesta en marcha del sGsss se crearon las Entidades Promotoras de Salud (EPS), 214 cuya función básica es la gestión del aseguramiento mediante la afiliación, el recaudo de las cotizaciones y la conformación de la red de prestación de servicios con Instituciones Prestadoras de Salud (IPS) de todos los niveles de atención, a través de las cuales las EPS deben garantizar el acceso a los servicios contenidos en el pos.

La afiliación al sGsss se puede hacer a través del régimen contributivo y subsidiado. Los afiliados al sistema mediante el régimen contributivo son las personas que realizan un aporte mensual denominado cotización, valor que se estima de acuerdo con su ingreso base, según lo definido en la ley. Son afiliados a él quienes se encuentren vinculados mediante contrato laboral, los pensionados y los trabajadores independientes con capacidad de pago. Por el contrario, los vinculados al sistema mediante el régimen subsidiado son personas sin capacidad de pago, que no pueden cubrir el monto total de la cotización mínima (5).

Adicionalmente, los afiliados y beneficiarios del sGsss, al momento de usar los servicios, están sujetos a cuotas moderadoras y pagos compartidos. Los primeros, con el objetivo de racionalizar el uso de los servicios del sistema, y los segundos, para complementar la financiación del POs (6).

Sin embargo, existen grupos poblacionales priorizados que quedan exentos de dichos pagos, con el fin de eliminar barreras de acceso para poblaciones vulnerables, dentro de los que se destacan las gestantes y los menores de un año de edad. De tal manera, el sGsss para las mujeres en estado de embarazo cubre los servicios de salud de control prenatal (CPN), atención del parto, control del posparto y la atención de las afectaciones relacionadas directamente con la lactancia, sin el cobro de pagos compartidos o cuotas moderadoras. 
De otra parte, la maternidad segura es una prioridad a nivel nacional. Es así como en el artículo 43 de la Carta Magna colombiana se establece que la mujer durante el embarazo y después del parto gozará de especial asistencia y protección del Estado. En desarrollo de este precepto constitucional, en la Ley 100 de 1993 se incluyó en el artículo 166 la forma en la que se desarrollará la protección y atención materna dentro del sGsss.

El artículo 166 de la Ley 100 de 1993 encuentra las bases de su implementación: primero, en la definición de los contenidos pos, cuyos últimos contenidos fueron establecidos en el Acuerdo 29 de la Comisión de Regulación en Salud (Cres); segundo, en la inclusión de la atención a las maternas dentro de las actividades de demanda inducida y de interés en salud pública; tercero, en la definición de una guía de atención específica definida en la Resolución 412 de 2000; y cuarto, en la definición del Plan Nacional de Salud Pública mediante el Decreto 3039 de 2007, el cual incluyó la atención materno-infantil dentro de las prioridades de salud pública del país.

Complementariamente, la Procuraduría General de la Nación, el Ministerio de Salud y Protección Social, la Superintendencia de Salud y el Instituto Nacional de Salud expidieron la Circular Externa ${ }^{\circ} 005$ de 2012 (7); con la implementación de esta, se pretende disminuir las muertes maternas y perinatales por causas médicas y no médicas prevenibles e intervenibles, con la eliminación total de las barreras de acceso dentro del sGsss para las maternas.

En adición, en el contexto internacional, la Organización de las Naciones Unidas (ONU) en el año 2000 incluyó en la Declaración del Milenio, como una de las metas que es necesario cumplir, la reducción de la mortalidad materno-infantil, estableciendo que sus países miembros deberían reducirla en tres cuartas partes para el año 2015 (8).

Sin embargo, a pesar de estos esfuerzos, el reporte del Fondo de Población de las Naciones Unidas (FPNU), el Fondo de las Naciones Unidas para la Infancia (Unicef), la Organización Mundial de la Salud (oMs) y el Banco Mundial, publicado en el 2012, mostró que a nivel mundial, entre 1990 y 2010, la tasa de mortalidad materna disminuyó en un $47 \%$ (9). Esto pone de manifiesto que es muy improbable que se alcance la meta de reducir la tasa mortalidad. Este reporte, adicionalmente, determinó algunas razones de peso para explicar la disminución de la mortalidad materna, entre las que se encuentran el uso de anticonceptivos, la atención del parto por personal de salud calificado y la implementación de programas de CPN.

A pesar de ello, es innegable que, exceptuando Sudáfrica, una reducción sustancial de las muertes maternas se ha logrado en todas las regiones del mundo. En el caso colombiano, dicha tasa ha disminuido de manera paulatina, ubicándose en 69,3 muertes maternas por cada 1000 nacido vivos en el año 2011 (10). El departamento del Magdalena supera, al igual que otras 18 entidades territoriales, el promedio nacional de mortalidad materna, al ubicarse por encima de 100 muertes maternas por cada 1000 nacidos vivos (10).

En cuanto al CPN, este es definido por la oMs como "serie de contactos, entrevistas, o visitas programadas de la embarazada con integrantes del equipo de salud, con el objetivo de vigilar la evolución del embarazo y obtener una adecuada preparación para el parto y la crianza" (11). Por ende, es un esfuerzo prioritario en el logro de este objetivo que los servicios de salud estén enfocados en la realización de controles prenatales y el desarrollo de guías de práctica clínica estandarizadas para estos. 
En particular, en Colombia, el Instituto Nacional de Salud encontró que el 34\% de los casos de muertes maternas tempranas están relacionados con la no asistencia a control prenatal (10), razón por la cual esta variable se constituye en un punto clave para que desde estrategias generadas por el sistema de salud y, en particular, implementadas por las IPS, se aporte a su disminución, impactando positivamente en la reducción de la mortalidad materna.

La investigación que se reporta en este artículo busca aportar proveyendo evidencia que soporte la toma de decisiones políticas encaminadas a reducir la inasistencia al CPN; para ello, el estudio se llevó a cabo en la ciudad de Santa Marta, capital costera del departamento del Magdalena localizado al norte de Colombia, que fue elevada al rango de Distrito Turístico Cultural e Histórico de la Nación (12).

Específicamente, la investigación tuvo lugar en ADM, IPs de salud de primer nivel de complejidad ubicada en el centro de la ciudad. Lo anterior, dado que ADM IPS es una institución enfocada en la Atención Primaria en Salud (APS), que ha implementado políticas y herramientas de atención preferencial para las maternas inscritas al CPN, en pro de garantizar: a) el acceso oportuno, mediante el sistema de recordación de consultas programadas y tiempo de asignación de cita menor a 24 horas (si es solicitada vía telefónica, correo electrónico, mensaje de texto) o asignación inmediata de solicitarse de forma presencial; b) continuidad en la atención a través del establecimiento de un grupo único de profesionales destinados a la atención del programa de CPN, encabezado por un especialista en ginecoobstetricia; y c) calidad en la prestación de servicios, realizando evaluaciones permanentes de la 216 adherencia a protocolos y guías de manejo de patologías propias del embarazo, así como de la gestión y resultados del programa de control prenatal institucional.

A partir de dichas evaluaciones de gestión y seguimiento del programa de CPN de ADM IPS surgieron los interrogantes que motivaron el presente estudio. Ante la evidencia de un porcentaje de inasistencia del $18 \%$, se realizaron los siguientes planteamientos: si el sGsss y la normativa vigente eliminan las barreras económicas de acceso dentro del sistema y, aunado a la eliminación activa de barreras de uso de los servicios de salud para maternas por parte de ADM IPS, ¿qué factores no contemplados hasta el momento por el sistema de salud colombiano podrían explicar la tendencia de inasistencia a citas programadas de controles prenatales por parte de las gestantes?, ¿cuál sería su peso en la problemática?, ¿existe relación directa entre dichos factores no contemplados por la norma y los desenlaces perinatales desfavorables?

De lo anterior parte la importancia de la caracterización de las diferentes barreras de acceso para intervenirlas y minimizar riesgo a la población de gestantes, en especial aquellas pertenecientes a grupos poblacionales vulnerables.

\section{Metodología}

Se realizó una investigación de diseño mixto, multietápico, de cohorte prospectivo, desarrollado durante un periodo total de observación de diez meses, comprendidos entre mayo de 2012 y febrero de 2013. La población objeto de la investigación fue la totalidad de las gestantes inscritas en el programa de Control Prenatal (49 pacientes), quienes asistieron a la consulta de medicina general en ADM IPS, localizada en la ciudad de Santa Marta, Colombia. La población no fue diferenciada por variables como edad, nivel 
socioeconómico, educativo o clasificación de riesgo gestacional. La investigación se dividió en tres etapas: detección del problema, revisión teórica, intervención y recolección de datos; las cuales se describen a continuación.

La primera etapa, denominada detección del problema, fue de tipo descriptivo y se desarrolló en tres instancias, a saber: dos de tipo cualitativo y una de tipo cuantitativo, cuyas fuentes de información fueron entrevistas con las directivas de ADM IPS, así como fuentes documentales y de registro de las bases de datos del sistema de información de la IPS y entrevistas con usuarias del servicio.

En primera instancia, se procedió a realizar un análisis de los datos recolectados mediante entrevistas en profundidad no estructuradas a las directivas, utilizando como instrumento de análisis el diagrama causa-efecto, con el propósito de determinar las principales variables que se asocian como posibles causas de inasistencia o cancelación de citas al CPN. Como resultado del análisis se identificaron a priori cinco grandes grupos de causas de inasistencias o cancelaciones de citas en la población objeto, a saber: usuario, EPS, personal asistencial, personal de atención al usuario y tecnología.

En segunda instancia, con base en los anteriores hallazgos, se procedió a identificar a todas las maternas inasistentes o que cancelaron citas de cPN. Para ello, se recolectó información contenida en la base de datos de asignación de citas de ADM IPS, denominada ruta de citas. En tercera instancia, con base en esta información, se procedió a contactar a las maternas vía telefónica, con el fin de aplicar un cuestionario que permitiera determinar el motivo de incumplimiento o postergación (ver anexo 1). Los resultados se analizaron mediante estadística descriptiva, específicamente con análisis de frecuencias y diagramación 20/80 o de Pareto.
En la segunda etapa, denominada intervención, con base en los resultados obtenidos en las fases anteriores, se implementó un plan de acción encaminado a mitigar el impacto de la principal causa identificada, a saber: barreras de acceso de tipo económico relacionadas con la imposibilidad de pagar el medio de trasporte utilizado para el desplazamiento desde el hogar hasta el centro médico y viceversa; el cual incluyó la búsqueda de apoyo económico a través de una fundación. Con tal propósito, se logró la participación de FuncoAndes, fundación que presta ayuda a mujeres cabeza de hogar y madres comunitarias en la ciudad de Santa Marta, a través de la entrega de subsidios de transporte a maternas del programa de control prenatal de ADM IPS.

Dicha entrega reconoció el monto que las gestantes pagaban por el medio de transporte urbano que empleaban para asistir al CPN, otorgándoles un subsidio por el valor de $\$$ 3000 pesos por gestante, una vez terminada la consulta. La duración de esta fase fue de cinco meses (octubre del 2012 a febrero del 2013), posteriormente a la cual se evaluó el impacto de la asignación de los subsidios, mediante la comparación del indicador de inasistencia del grupo de estudio antes y después de la intervención.

En la tercera etapa, conocimiento de determinantes sociales, con el fin de buscar posibles asociaciones entre los datos de determinantes sociales con complicaciones maternas perinatales, se realizó una encuesta telefónica adicional a todas las pacientes gestantes, con el fin de obtener información acerca de los determinantes sociales de la salud (ver anexo 2), tales como: nivel socioeconómico y educativo, estructura familiar, localización geográfica de la vivienda y distancia y tiempo empleado en el desplazamiento desde sus hogares hasta la IPS. Esta información se analizó con la obtenida en la 
Fredy Guillermo Rodríguez-PÁez • Wilson Giovanni Jiménez-Barbosa • Cindy Alejandra

Jiménez-González • Ángela Edith Coral-Córdoba • Paola Carolina Ramírez-Solano • Néstor

Raúl Ramos-Navas

base de datos de hospitalizaciones de pacientes, mediante análisis bivariado utilizando tablas de $2 \times 2$.

\section{Resultados}

\section{Primera etapa}

La población asignada a ADM IPS corresponde a 1217 usuarios, de los cuales 632 son mujeres $(51,9 \%)$. De ellas, $337(53,3 \%)$ se encuentran en edad fértil (15-45 años). En cuanto a la caracterización socioeconómica del total de la población, se encuentra que el 97\% devenga menos de dos salarios mínimos mensuales legales vigentes (SMMLV), ${ }^{1}$ el $2 \%$ entre dos y cinco SMMLV y el $1 \%$ restante más de cinco SMmLv (6). El 99,4\% de la población vive en el área urbana y el restante $0,6 \%$ en el área rural. Las viviendas de la totalidad de la población se ubican en barrios estratificados ${ }^{2}$ en nivel socioeconómico 1, 2 y 3 (13), las que cuentan con servicios públicos básicos: energía eléctrica, provisión de agua potable, gas y alcantarillado; el $80 \%$ viven en áreas con vías pavimentadas.

En cuanto a la inscripción al programa de CPN, durante el periodo de estudio, el total de participantes fue de 49 pacientes gestantes, cuyas características generales se presentan en la tabla 1.

1 El smmLv en Colombia para el año 2013 corresponde a COP $\$ 589500$, lo que equivale a USD $\$ 310$, teniendo como tasa de cambio un valor promedio de COP \$ 1900 por USD.

2 En Colombia, los inmuebles residenciales (viviendas) son estratificados de acuerdo con metodologías definidas por el Gobierno Nacional, que según lo definido en el artículo 102 de la Ley 142 de 1994, modificado por el artículo 16 de la Ley 689 de 2001, se clasifican máximo en seis estratos socioeconómicos: 1) bajo-bajo, 2) bajo, 3) medio-bajo, 4) medio, 5) medio-alto y 6) alto, dependiendo de las características particulares de los municipios y distritos.
El rango de edad de las maternas que asistieron al CPN osciló entre 16 y 39 años. La edad promedio de las gestantes fue de 26,9 años. Por grupos etarios, la mayor participación fue del grupo de 21 a 25 años, con $30 \%$, seguido por el grupo de 26 a 30 años, con $22 \%$. Dentro del grupo estudiado no se encontraron gestantes menores de 16 años ni mayores de 40 años.

De las cinco categorías planteadas en el análisis causa-efecto (personal asistencial, personal SIAU, EPS, usuario y tecnología), se encontró que la totalidad de las causas que aducían las gestantes para la inasistencia o postergación de citas a control prenatal se enmarcaban dentro de las categorías usuario y EPS (figura 1).

Dentro de la categoría usuario, las causas estaban asociadas, principalmente, a la subcategorías económica (dificultad de pago de medios de transporte o no pago oportuno de la cotización, por lo que la EPs no emitía la autorización), y en la de EPS, por retraso en la autorización y proceso de autorización, realización y entrega de resultados de paraclínicos.

Al realizar el análisis de las causales de inasistencia al CPN referidas en la tabla 3, se encontró que las económicas, en particular las relacionadas con la tenencia de recursos que les permitiera el pago de medios de transporte a las maternas, representaron un $43 \%$, constituyéndose en la principal barrera de acceso.

\section{Segunda etapa}

Con base en los resultados de la primera etapa, se procedió a llevar a cabo la segunda etapa de la investigación, denominada: intervención. Para tal fin se contactó a la Fundación FuncoAndes, quienes dieron el patrocinio económico para el otorgamiento 
TABLA 1. Características población

\begin{tabular}{|c|c|c|}
\hline Total población estudiada & \multicolumn{2}{|c|}{49 pacientes gestantes } \\
\hline \multicolumn{3}{|l|}{ Edad (en años) } \\
\hline Rango de edad & \multicolumn{2}{|c|}{$16-39$} \\
\hline Edad promedio & \multicolumn{2}{|c|}{26,9} \\
\hline \multirow{5}{*}{ Frecuencia de distribución } & $16-20$ & 9 \\
\hline & $21-25$ & 15 \\
\hline & $26-30$ & 11 \\
\hline & $31-35$ & 7 \\
\hline & $36-40$ & 7 \\
\hline \multicolumn{3}{|c|}{ Clasificación de riesgo escala biopsicosocial } \\
\hline$\leq 2$ & \multicolumn{2}{|c|}{21} \\
\hline$\geq 3$ & \multicolumn{2}{|c|}{28} \\
\hline \multicolumn{3}{|l|}{ Nivel socioeconómico } \\
\hline Estrato 1 & \multicolumn{2}{|c|}{32} \\
\hline Estrato 2 & \multicolumn{2}{|c|}{17} \\
\hline \multicolumn{3}{|l|}{ Número de hijos } \\
\hline Promedio & \multicolumn{2}{|c|}{2,59} \\
\hline Rango numero hijos & \multicolumn{2}{|c|}{$1-8$} \\
\hline \multicolumn{3}{|l|}{ Nivel educativo } \\
\hline Analfabeta & \multicolumn{2}{|c|}{1} \\
\hline Primaria incompleta & \multicolumn{2}{|c|}{1} \\
\hline Primaria completa & \multicolumn{2}{|c|}{6} \\
\hline Secundaria incompleta & \multicolumn{2}{|c|}{10} \\
\hline Secundaria completa & \multicolumn{2}{|c|}{24} \\
\hline Título técnico laboral & \multicolumn{2}{|c|}{6} \\
\hline Título universitario & \multicolumn{2}{|c|}{1} \\
\hline \multicolumn{3}{|l|}{ Actividad laboral } \\
\hline Hogar & \multicolumn{2}{|c|}{36} \\
\hline Servicios generales & \multicolumn{2}{|c|}{5} \\
\hline comercio & \multicolumn{2}{|c|}{7} \\
\hline otros & \multicolumn{2}{|c|}{1} \\
\hline \multicolumn{3}{|l|}{ Generación de ingresos en núcleo familiar } \\
\hline Aportan como madres solteras & \multicolumn{2}{|c|}{12} \\
\hline Aporta cónyuge & \multicolumn{2}{|c|}{25} \\
\hline Tanto cónyuge como gestante aportan & & \\
\hline
\end{tabular}

Fuente: elaboración propia 
Fredy Guillermo Rodríguez-PÁez • Wilson Giovanni Jiménez-Barbosa • Cindy Alejandra Jiménez-González • Ángela Edith Coral-Córdoba • Paola Carolina Ramírez-Solano • Néstor

RaÚl RAMOS-NAVAS

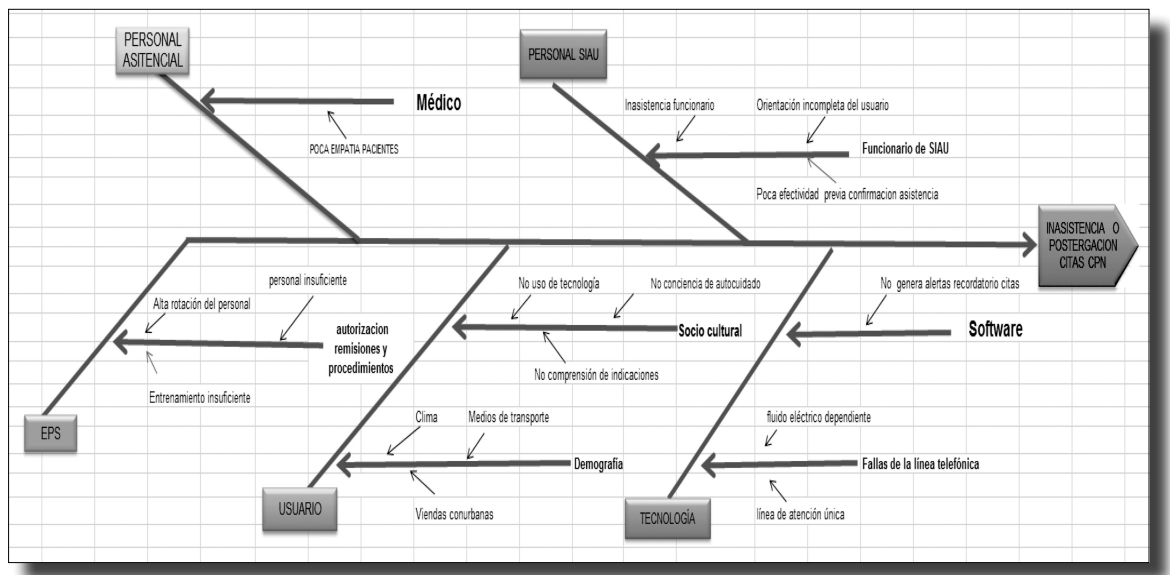

Figura 1. Análisis a priori de Causas de inasistencia a Citas de CONTROL PRenatal por medicina general Fuente: elaboración propia

de subsidios de transporte a las pacientes gestantes inscritas en el programa de CPN de ADM IPS.

Como resultado del otorgamiento de subsidios de transporte a las maternas para que pudiesen asistir al CPN, se logró una reducción ostensible de la inasistencia, al pasar de un $17,5 \%$ para la etapa preintervención, a un $2,3 \%$ en la etapa de intervención, es decir, se presentó una reducción de 15,2 puntos porcentuales, al comparar las dos fases (tabla 2).

\section{Tercera etapa}

A continuación de la etapa de intervención, se aplicó una segunda encuesta, obteniéndose información referente a los determinantes sociales de la población objeto de estudio, (tabla 4).

TABLA 2. CPN PROGRAMADOS E INASISTENCIAS MENSUALES ADM IPS

\begin{tabular}{|l|l|l|l|}
\hline Fase & Mes & $\mathbf{N}^{\circ}$ cpn programados & Inasistencias \\
\hline \multirow{4}{*}{$\begin{array}{l}\text { Primera fase: detec- } \\
\text { ción del problema }\end{array}$} & Mayo & 11 & 3 \\
\cline { 2 - 4 } & Junio & 17 & 2 \\
\cline { 2 - 4 } & Aglio & 47 & 12 \\
\cline { 2 - 4 } & Septiembre & 21 & 2 \\
\cline { 2 - 4 } & Total & 24 & 2 \\
\hline \multirow{4}{*}{$\begin{array}{l}\text { Segunda fase: inter- } \\
\text { vención }\end{array}$} & Octubre & 120 & 21 \\
\cline { 2 - 4 } & Noviembre & 29 & 0 \\
\cline { 2 - 4 } & Diciembre & 14 & 2 \\
\cline { 2 - 4 } & Enero & 12 & 0 \\
\cline { 2 - 4 } & Febrero & 13 & 2 \\
\cline { 2 - 4 } & Total & 85 & 0 \\
\hline
\end{tabular}

Fuente: elaboración propia 
TABLA 3. Causales inasistencia o Cancelación Citas control prenatal fase 1

\begin{tabular}{|c|c|c|c|}
\hline \multicolumn{2}{|l|}{ Barrera de acceso encontrada } & Frecuencia & Porcentaje \\
\hline 1 & Económica, dificultad pago de medios de transporte & 9 & 15 \\
\hline 2 & Retraso en la autorización y realización paraclínicos & 5 & 8 \\
\hline 3 & No datos & 2 & 3 \\
\hline 4 & Climatológica & 2 & 3 \\
\hline 5 & Económica, no pago oportuno cotización & 1 & 2 \\
\hline 6 & Fortuito & 1 & 2 \\
\hline 7 & Geográfica & 1 & 2 \\
\hline & Total inasistencias & 21 & 18 \\
\hline \multicolumn{2}{r}{ Total de asistencias } & 99 & 82 \\
\hline & & 120 & 100 \\
\hline
\end{tabular}

Fuente: elaboración propia

En cuanto al nivel socioeconómico, el $65 \%$ de las gestantes vivían en casas estratificadas en nivel 1, que corresponde al grupo denominado bajo-bajo. El restante $35 \%$ se ubicó en estrato 2, grupo denominado bajo. Por tanto, se concluye que las maternas que asistieron al programa de CPN viven en barrios pobres de la ciudad.

TABLA 4. Evaluación de determinantes SOCIALES de LA SALUd EN PACIENTES GESTANTES de La IPS ADM. MAYO DEL 2012 A FEBRERO DEL 2013

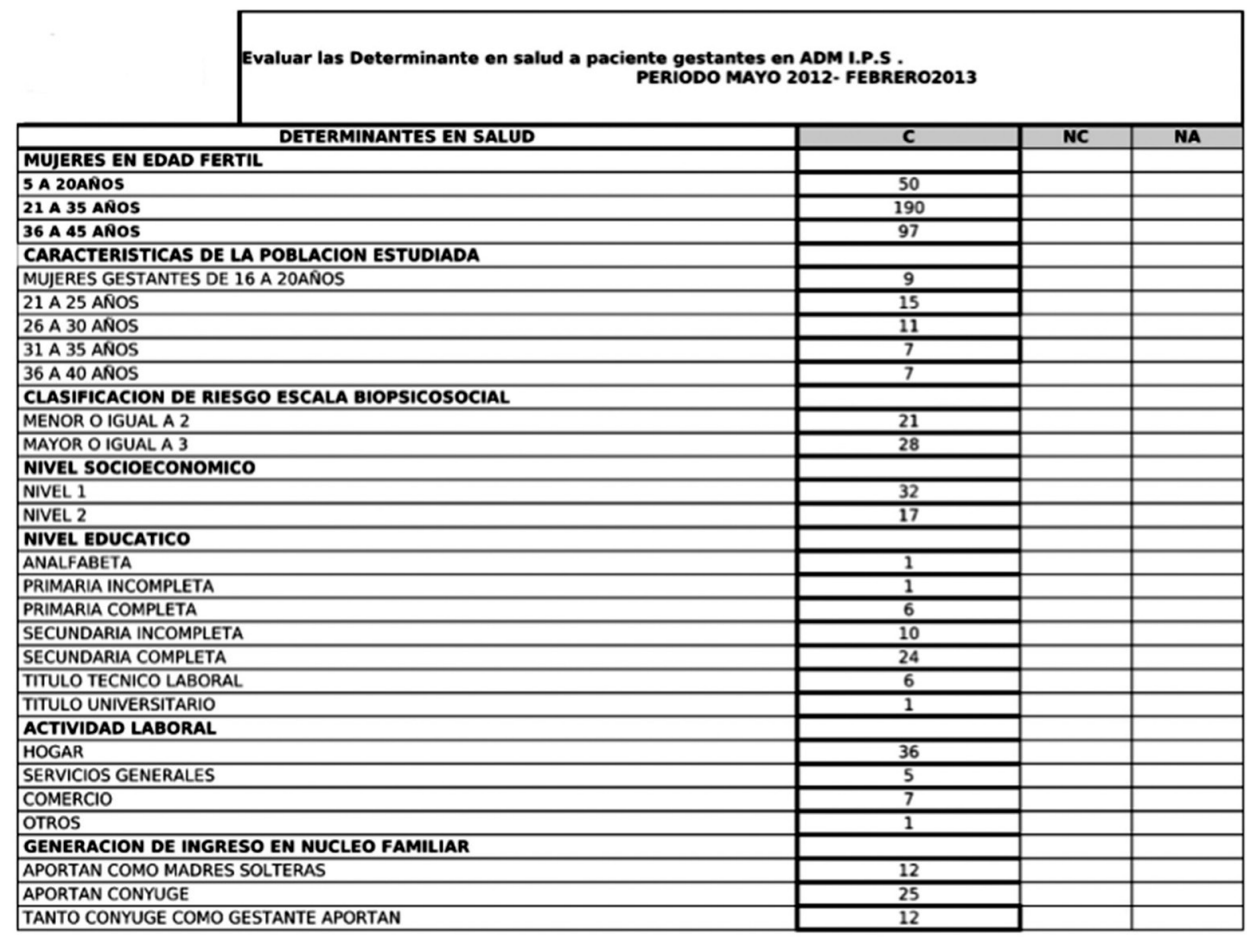

Fuente: IPS ADM de Santa Marta 
Fredy Guillermo Rodríguez-Páez • Wilson Giovanni Jiménez-Barbosa • Cindy Alejandra Jiménez-González • Ángela Edith Coral-Córdoba • Paola Carolina Ramírez-Solano • Néstor

Raúl Ramos-Navas

En cuanto al número de hijos que ya tenían las gestantes, se encontró que variaba en un rango de 1 a 8 , ubicándose el promedio en 2,59, que resulta inferior al valor de 3,5 hijos por mujer identificado para el departamento del Magdalena por la Encuesta de Demografía y Salud realizada por Profamilia (14). Sin embargo, este resultado es superior al promedio nacional de 2,1 hijos por mujer identificado en el mismo estudio.

$\mathrm{Al}$ analizar el nivel educativo de la gestantes, se encontró que el $49 \%$ había completado la educación secundaria, el 20\% cursó algunos grados de secundaria sin completarla, el $12 \%$ la primaria completa, mientras que el grado de técnico laboral lo alcanzó otro $12 \%$. Vale la pena destacar que el $2 \%$ era analfabeta.

Adicionalmente, utilizando el sistema de información geográfica Google Earth (15), se establecieron las distancias de los trayectos trazados entre ADM IPS y direcciones de las maternas. De esta forma, se identificaron dos grupos: uno, conformado por gestantes con viviendas ubicadas a más de $10 \mathrm{~km}(52 \%)$, y un segundo constituido por aquellas que habitan a menos de $10 \mathrm{~km}(48 \%)$. La distribución espacial de las maternas se puede observar con mayor claridad en la figura 2.

Finalmente, de las historias clínicas de las gestantes se tomaron los datos de la escala de clasificación de riesgo biopsicosocial (tabla 5) (16), con el fin de diferenciar las pacientes con diagnóstico de alto riesgo obstétrico o riesgo bajo. Se tomó como punto de corte tres o más factores de riesgo, presentes para clasificar el alto riesgo obstétrico, según lo estipulado en la guía de atención prenatal de la Resolución 412 de 2000 (17).

Esta información fue comparada con la base de datos de hospitalizaciones de gestantes y neonatos acaecidas durante todo el periodo de estudio, utilizando análisis bivariado con tablas de $2 \times 2$, a fin de asociar las variables sociales con factor de riesgo obstétrico.

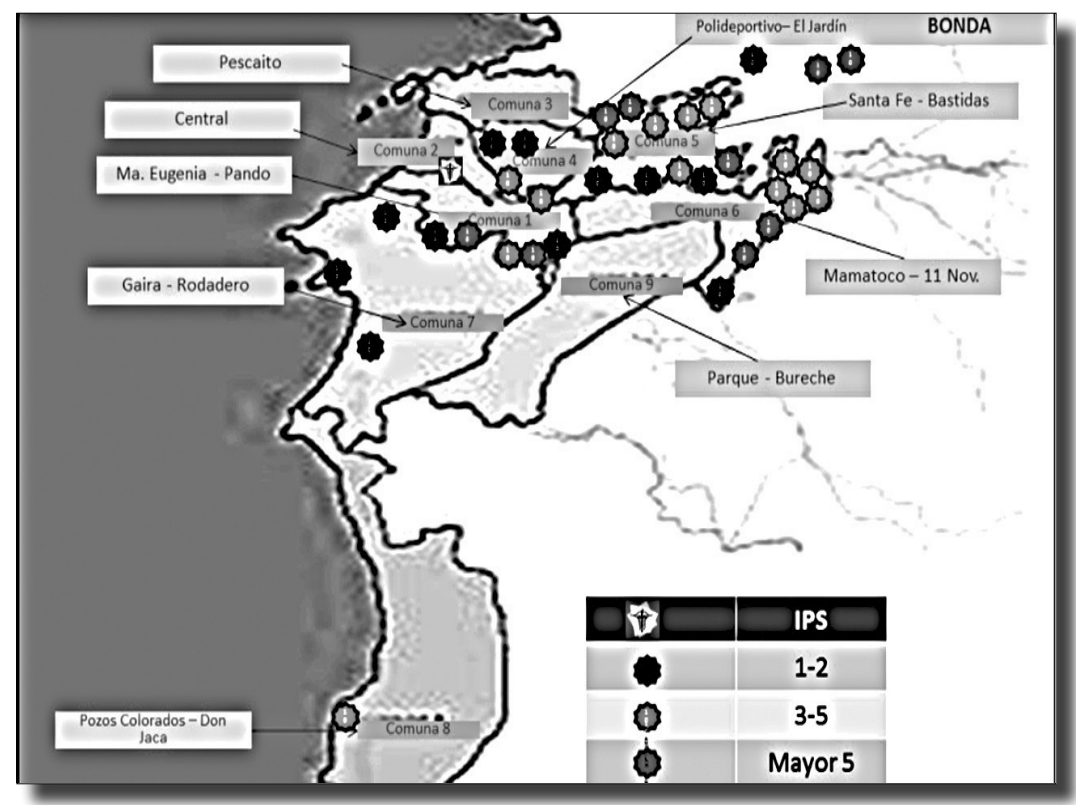

Figura 2. Distribución geográfica de hogares, MATERnas E IPS DE CPN Distribuidas POR NIVELES DE RIESGO Fuente: elaboración propia BIOPSICOSOCIAL 
TABLA 5. ESCALA DE RIESGO BIOPSICOSOCIAL PRENATAL

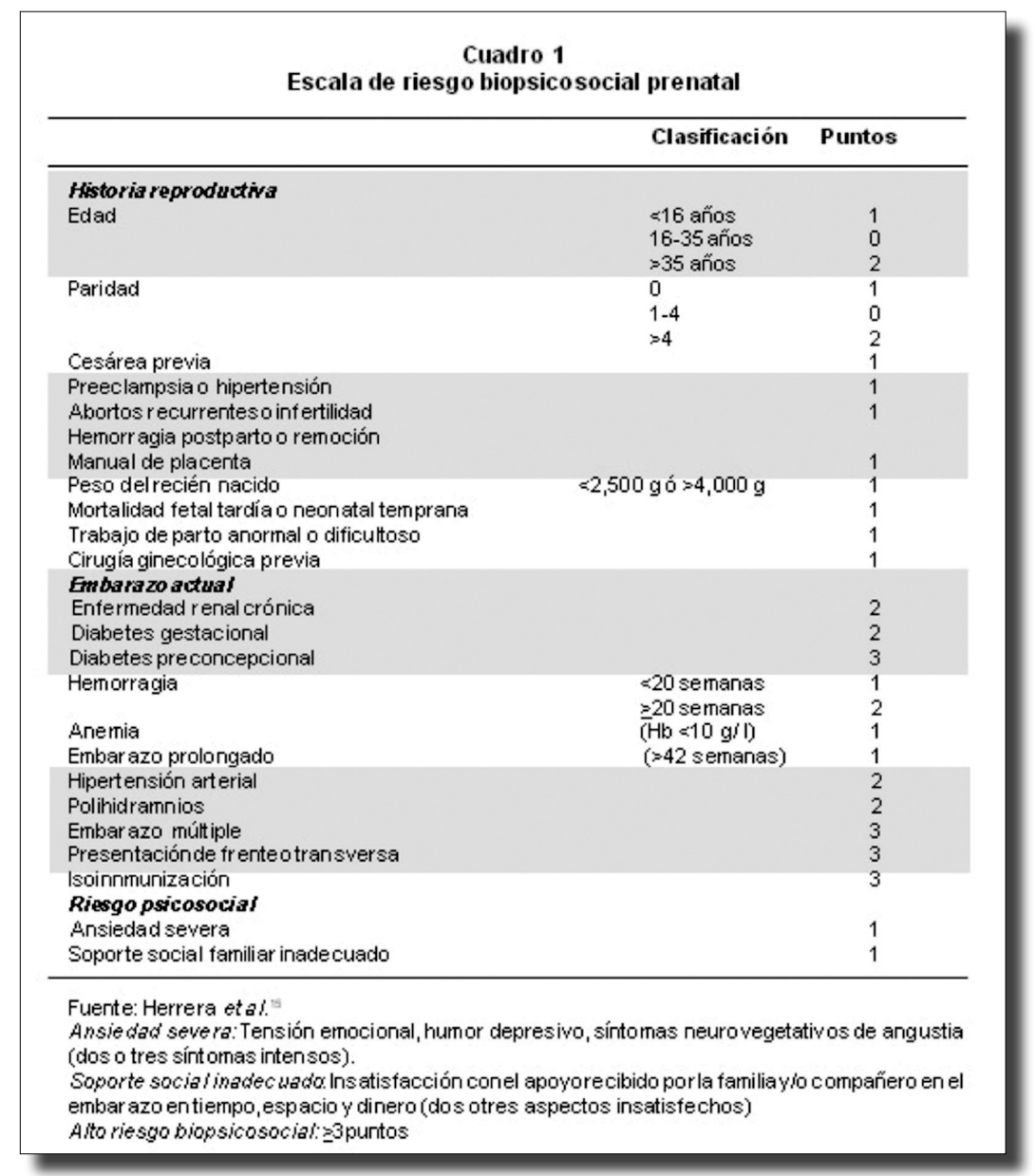

Como resultado, se encontró que la clasificación de alto riesgo obstétrico se relaciona de manera directa con complicaciones maternas perinatales y complicaciones neonatales, con una tasa de incidencia de 36 y $64 \%$, respectivamente, versus cero casos para aquellas clasificadas como bajo riesgo, con un riesgo atribuible porcentual del $100 \%$ para ambos.

En cuanto al nivel socioeconómico bajo (estrato 1), con un riesgo relativo (RR) de 2,6, también mostró relación con la presentación de complicaciones maternas, con una tasa de incidencia de hospitalizaciones del $33 \%$, comparado con un $13 \%$ para aquellas maternas en estratos 2 y superiores.
De igual forma, una distancia mayor de 10 $\mathrm{km}$ entre la vivienda de la gestante y la IPs se relacionó con un riesgo mayor de desenlaces maternos perinatales negativos, con una tasa de incidencia de $37 \%$, versus $11 \%$ para aquellas que viven a menos de $10 \mathrm{~km}$ de distancia de la IPS, con un riesgo porcentual atribuible de $70 \%$ y un RR de 3 .

En contraposición, características como educación superior y hogares donde la responsabilidad económica recae sobre el hombre, se comportaron como factor protector. En el primero, educación universitaria o técnica, comparado con aquellas maternas con educación básica primaria o máximo secundaria, 
no se presentó ningún caso de complicación materna ni neonatal. En el segundo, hogares donde la carga económica está a cargo del hombre, se evidenció una tasa de incidencia en hospitalizaciones durante la gestación de $29 \%$, comparado con aquellos hogares donde la responsabilidad económica está a cargo de la gestante o tienen gastos compartidos, con una tasa de incidencia del $44 \%$.

\section{Discusión}

Múltiples estudios clínicos alrededor del mundo han tratado de demostrar la asociación directa entre el riesgo materno-fetal y la implementación de programas de CPN de calidad que procuren una adecuada utilización de la atención prenatal. Los hallazgos sugieren que la utilización inadecuada de atención prenatal se produce con más frecuencia en mujeres de grupos de riesgo como: menores de 20 años, bajo estrato socioeconómico, bajo nivel de escolaridad y minoría racial, asociándose a una atención inadecuada con mayor riesgo de prematuridad, muerte fetal, muerte neonatal tardía y muerte materna (18). Los hallazgos de la investigación se orientan hacia el mismo tipo de resultado, al destacarse que factores tales como el bajo nivel socioeconómico, la distancia del lugar de residencia al prestador, el bajo nivel educativo y ser la gestante cabeza de familia, se asocian con aumento en el riesgo perinatal.

Es así como "En respuesta a la creciente preocupación suscitada por las persistentes inequidades las cuales son cada vez mayores, la Organización Mundial de la Salud estableció en 2005 la Comisión sobre Determinantes Sociales de la Salud, para que ofreciera asesoramiento respecto a la manera de mitigarlas" (19). Determinantes sociales de la salud, definidos como el "conjunto de factores personales, sociales, políticos y ambientales que determinan el estado de salud de los individuos y las poblaciones" (20). Los determinantes sociales de la salud son las circunstancias en que las personas nacen, crecen, viven, trabajan y envejecen, incluido el sistema de salud. Esas circunstancias son el resultado de la distribución del dinero, el poder y los recursos a nivel mundial, nacional y local, que a su vez depende de las políticas adoptadas (21).

La importancia del análisis de los determinantes sociales de la salud radica en la relación directa de estos con el estado de salud de una población, relacionándose de manera inversa con las inequidades sanitarias, lo que explica las situaciones y los comportamientos relacionados con la salud de las comunidades. Por ello, en el informe final de la Comisión, publicado en agosto del 2008, se proponen tres recomendaciones generales: mejorar las condiciones de vida cotidianas, luchar contra la distribución desigual del poder, el dinero y los recursos, y la medición y análisis del problema (22).

Un estudio realizado en Estados Unidos, donde durante el posparto se realizaron entrevistas a mujeres que ingresaron a través de los servicios de urgencias sin ningún control prenatal en ocho hospitales de Sacramento, California durante dos meses en el 2012, Aved BM et ál. encontraron que las principales barreras de acceso a las que hicieron referencia las pacientes fueron las dificultades de transporte, clasificándolas, al igual que en el presente estudio, como la principal causa por la cual las mujeres nunca trataron de obtener atención médica, afirmación que se ve ratificada en la investigación, al establecer que la principal barrera de acceso que enfrentan las gestantes para la asistencia al CPN es la de no contar con recursos económicos que les permitan sufragar los costos de trasporte desde su hogar hacia la IPS y viceversa. 
Desde el punto de vista médico, se citaron dificultades administrativas, requisitos adicionales de atención y bajos ingresos de las pacientes como barreras para el cuidado de esta población (23). Esto coincide con la disminución del $87 \%$ de inasistencia tras eliminar la barrera de acceso económica a través de la entrega de subsidios de transporte.

En Zambia, en el 2011, se evaluó cómo la distancia a las instalaciones de los servicios de salud y el nivel de prestación de servicios de estas instituciones, donde se llevaban a cabo los controles prenatales, influyó en el número y el momento de las visitas prenatales y la calidad de la atención recibida. El estudio encontró que por cada aumento de $10 \mathrm{~km}$ de distancia, las probabilidades de que las mujeres reciban buena calidad en el control pretal disminuye en un $25 \%$, mientras que cada aumento en el nivel de categoría de disposición de la instalación más cercana se asoció con un aumento del $54 \%$ en las probabilidades de recepción de buena calidad en la atención prenatal (24).

En concordancia con lo hallado en Zambia, el presente estudio encontró que las maternas cuyo hogar se encuentra a más de 10 $\mathrm{km}$ de distancia, presentaron una tasa de incidencia de complicaciones maternas perinatales $26 \%$ mayor, comparado con aquellas que viven a una distancia menor de $10 \mathrm{~km}$ respecto a la IPS donde asistían a su CPN.

Sin embargo, cabe anotar que aun cuando la identificación y la eliminación de la barreras de acceso fácilmente identificables resultan per se en una mejora en los resultados perinatales materno-fetales, "[...] la activa eliminación de las barreras en la atención no garantiza la participación a largo plazo y coherente de las mujeres vulnerables en la atención preventiva. Hay factores desconocidos más allá de las barreras conocidas que afectan la participación en el cuidado preconcepcional” (25).

Por ello, se buscó determinar las expectativas de las gestantes frente al control prenatal de calidad, partiendo de las tres principales categorías y los subtemas establecidas por el modelo de Donabedian sobre la atención de salud de calidad, a saber: estructura de la atención, procesos de atención clínica y procesos interpersonales de la atención. Como resultado, la calidad de la atención prenatal fue asociada por las maternas con la existencia de buenas relaciones interpersonales. Esta categoría es importante, ya que tiene un papel en la mitigación de efectos adversos, al promover la participación de las mujeres en su propio cuidado y su adhesión al control prenatal.

Los anteriores resultados son coincidentes con los principales hallazgos de la presente investigación, lo que hace pensar que la calidad de la atención del CPN requiere intervenciones específicas que trasciendan el ámbito clínico para articularse con componentes psicosociales que afectan la asistencia de las gestantes al programa, máxime en comunidades con condiciones socioeconómicas asociadas a la pobreza, independientemente de que estén afiliadas al régimen contributivo o al subsidiado.

\section{Conclusiones}

La primera etapa permitió identificar que la principal causa de inasistencia al CPN fue la presencia de barreras de acceso de tipo económico, asociadas a la condición socioeconómica de las gestantes, lo que les generaba dificultades en el pago de medios de transporte para asistir a los CPN programados. Dicha barrera fue resuelta en la segunda etapa, a través de la entrega de subsidios económicos obtenidos mediante 
gestión realizada por la IPS ante la Fundación FuncoAndes, lo que impactó positivamente en la reducción de la inasistencia al CPN.

La tercera etapa puso en evidencia que entre las gestantes estudiadas, las características poblacionales relacionadas con mayor número de hospitalizaciones durante la gestación, con complicaciones perinatales maternas y neonatales, fueron las siguientes: tener clasificación de alto riesgo obstétrico, pertenecer a un estrato socioeconómico bajo y habitar a más de $10 \mathrm{~km}$ de distancia respecto a la IPS. Estos hallazgos son similares a los reportados en la literatura. Adicionalmente, se observó que factores como la educación superior y el sustento económico a cargo de hombre cabeza de familia se comportan como características reductoras de riesgo.

Como conclusión general, la investigación hizo evidente que la inasistencia a los CPN no solo está asociada a la disponibilidad de infraestructura de salud, sino que adicionalmente, está relacionada con la presencia de barreras económicas, socioculturales y geográficas que en muchos casos no están incluidas dentro de las políticas y estrategias de los sistemas de salud. Por ello, se hace necesario que los sistemas de salud tengan un enfoque inter y transectorial, donde la estrategia de APS puede ser el camino para eliminar las dificultades que limitan el acceso efectivo a los servicios de salud, en este caso en particular, del CPN.

Por tanto, las iniciativas de salud pública para garantizar el acceso oportuno y adecuado a los sistemas de salud deberían incluir evaluaciones individualizadas de las poblaciones que es preciso atender, con el propósito de identificar determinantes sociales de la salud que permitan, a través de planes de acción inter y transectoriales, prevenir, mitigar y/o superar las desigualdades que ponen sistemáticamente en desventaja a grupos poblacio- nales vulnerables, con lo cual se alcanzaría un mayor nivel de equidad en salud y, por ende, de justicia social.

\section{Referencias bibliográficas}

1. Constitución Política de Colombia de 1991.

2. Corte Constitucional de Colombia. Sentencia T-760, Derecho a la salud, 31 de julio del 2008.

3. Vargas I, Vázquez M, Jané E. Equidad y reformas de los sistemas de salud en Latinoamérica. Cad. Saúde Pública [Serie en Internet]. 2002, [aprox. 10 páginas] Disponible en: http://dx.doi.org/10.1590/ S0102-311X2002000400003

4. Céspedes J, Jaramillo I, Martínez R, Olaya S, Reynales J, Uribe C et ál. Efectos de la Reforma de la Seguridad Social en Salud en Colombia sobre la equidad en el acceso y la utilización de servicios de salud. Rev Salud Pública. 2000 Julio; 2 (2): 145-64.

5. Congreso de la República de Colombia. Sistema de seguridad social integral. Ley 100. Pub. Diario oficial 41148 (Dic 23, 1993).

6. Consejo Nacional de Seguridad Social en Salud. Régimen de Pagos Compartidos y Cuotas moderadoras. Acuerdo 0260 Pub. Diario Oficial 45474 (Feb 27, 2004).

7. Procuraduría General de La Nación, Ministerio de Salud y Protección Social, Superintendencia Nacional de Salud. Instituto Nacional de Salud. Intensificación de acciones para garantizar la maternidad segura a nivel Nacional. Circular Conjunta Externa No. 005. (Feb 27, 2001).

8. Organización de las Naciones Unidas (ONU), Asamblea General. Declaración del Milenio. 2000 Septiembre 13

9. Fondo de Población de las Naciones Unidas (FPNU), Fondo de las Naciones Unidas para la Infancia (Unicef), Organización Mundial de la Salud (oms), Banco Mundial. Trends in Maternal Mortality: 1990 to 2010. 2012. Library Cataloguing-in-Publication Data. 1-44.

10. Cuevas E. Informe del evento mortalidad materna hasta el periodo epidemiológico 6, Colombia, 2014. versión 02. Instituto Nacional de Salud. 2014.

11. ops, oms. Salud perinatal. Boletín del Centro Latinoamericano de Perinatología y Desarrollo Humano. 1990; 3 (10): 1-10.

12. Alcaldía Distrital de Santa Marta. Plan Distrital de Salud Pública 2008-2011. Gaceta Distrital. 2008 sep. 28; 38.

13. Colombia, Congreso de la República. Régimen de Actos y Contratos de las Empresas, Ley 681. Diario Oficial. 2001; ago. 28, 44537.

14. Profamilia. Encuesta Nacional de Demografía y Salud. 2010

15. Google Earth [Serie en Internet] 1998 [consultado el 15 de marzo del 2013]. Disponible en: http://www. google.com/intl/es/earth/index.html.

16. Herrera J. Aplicación de un modelo biopsicosocial para la reducción de la morbilidad y mortalidad 
materna y perinatal en Colombia. Cali: Universidad del Valle; 2001.

17. Colombia, Ministerio de Salud. Actividades, procedimientos e intervenciones. Res. $n^{\circ} 412$ (Feb 25, 2000).

18. Partridge S, Balayla J, Holcroft C, Abenhaim H. Inadequate Prenatal Care Utilization and Risks of Infant Mortality and Poor Birth Outcome: A Retrospective Analysis of 28,729,765 U.S. Deliveries over 8 Years. American Journal of Perinatology. 2012 julio 26; 29 (10): 787-94.

19. Organización Mundial de la Salud (oms). Reducir las inequidades sanitarias actuando sobre los determinantes sociales de la salud. La 62 ${ }^{\mathrm{a}}$ Asamblea Mundial de la Salud, Octava sesión plenaria [Serie en Internet] 2009 [consultado el 15 de marzo del 2013]. Disponible en: http://www.who.int/social_determinants/es/

20. Organización Mundial de la Salud (oms). Glosario de promoción de la salud [Serie en Internet] 1998 [consutado el 14 de marzo del 2013]. Disponible en: http:// apps.who.int/gb/archive/pdf_files/WHA54/sa548.pdf
21. Organización Mundial de la Salud (oms). Determinantes sociales de la salud [Serie en Internet], 21 de octubre de 2011 [consultado el 14 de marzo del 2013]. Disponible en: http://www.who.int/topics/ social_determinants/es/index.html

22. Organización Mundial de la Salud (oms), Comisión Sobre Determinantes Sociales de la Salud. Subsanar las desigualdades en una generación. Informe final [Serie en Internet], 2008 [consultado el 13 de marzo del 2013]. Disponible en: http://whqlibdoc. who.int/publications/2009/9789243563701_spa.pdf

23. Aved BM, Irwin MM, Cummings LS, Findeisen N. Barriers to Prenatal Care for Low-Income Women. West J Med. 1993 Mayo; 158 (5): 493-98.

24. Kyei, Campbell, Gabrysch. The Influence of Distance and Level of Service Provision on Antenatal Care Use in Rural Zambia. PLoS One - Public Library of Science. 2012 Octubre 4; 7 (10): 1-6.

25. Donabedian, A. La calidad de la atención médica, definición y métodos de evaluación. México: Editorial La Prensa Médica Mexicana; 1984.

\section{ANEXOS}

Anexo 1. Encuesta de inasistencia al usuario de consulta especializada en la ips adm de Santa Marta

\begin{tabular}{|c|c|c|c|c|c|}
\hline & \multicolumn{4}{|c|}{$\begin{array}{l}\text { Evaluar las principales causas de inasistencia por factores atribuibles al usuario a consulta especializada en ADM } \\
\text { IPS. }\end{array}$} & \multirow[t]{2}{*}{ PERIODO ABRIL-MAYO 2013} \\
\hline \multirow{2}{*}{\multicolumn{6}{|c|}{$\begin{array}{l}\text { CRITERRO, PROCEDIMEIINTOS Y/O ACTIVIDADES DE CONTROL PRENATAL } \\
\text { El Usuario estála inscrito en los programas que brinda AD.M- L.PS }\end{array}$}} \\
\hline & & & & & \\
\hline \multirow{2}{*}{\multicolumn{6}{|c|}{ Hubo verificación de atos en cuantolnombre,cédula,atelefono,dirreccion de residencia.etc) }} \\
\hline \multicolumn{5}{|c|}{ 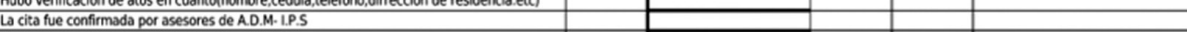 } & \\
\hline \multicolumn{6}{|c|}{ Se dio cita oportuna en el momento de solicitarla } \\
\hline \multicolumn{6}{|c|}{ Hay descripción completa hacerca de el dia y hora de su cita asignada } \\
\hline \multirow{2}{*}{\multicolumn{6}{|c|}{$\begin{array}{l}\text { A no poder asistir a su cita asignadá, le dieron la opción de cancelarla } \\
\text { Dieron a conocer los medios de comunicación, para cancelación de citas }\end{array}$}} \\
\hline \multirow{2}{*}{\multicolumn{6}{|c|}{$\begin{array}{l}\text { Dieron a conocer los medios de comunicación, para canceleción de citas } \\
\text { Si cancelo su cita, dio a conocer la causa de inasistencia }\end{array}$}} \\
\hline & & & & & \\
\hline \multicolumn{6}{|c|}{ La inasistencia fue por; medios de transporte? } \\
\hline \multicolumn{6}{|c|}{ Condiciones climaticos } \\
\hline \multicolumn{6}{|c|}{ Sul lugar de residencia esta a una distancia signficcativa a $A D . M . P . P S$} \\
\hline \multicolumn{6}{|c|}{ Conoce y Uuiliza los medios para cancelación de citas } \\
\hline \multicolumn{6}{|c|}{ 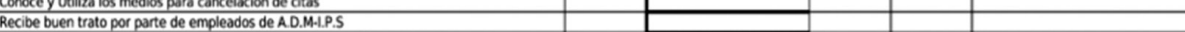 } \\
\hline El usuario ha presentado a & guna inconformidad con los servicios prestados & & & & \\
\hline
\end{tabular}

Fuente: IPS ADM de Santa Marta 